CRYSTALLOGRAPHIC COMMUNICATIONS

ISSN 2056-9890

Received 19 May 2020

Accepted 25 May 2020

Edited by A. V. Yatsenko, Moscow State University, Russia

Keywords: crystal structure; hydrogen bonding; cycloaddition products; $1 \mathrm{H}$-tetrazole-5-thione ring; $\pi-\pi$ stacking interactions.

CCDC reference: 2005595

Supporting information: this article has supporting information at journals.iucr.org/e

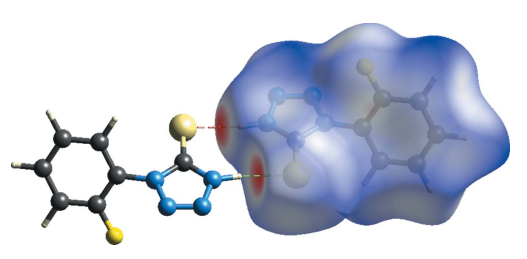

OPEN $\partial$ ACCESS

\section{Crystal structure and Hirshfeld surface analysis of 1-(2-fluorophenyl)-1H-tetrazole-5(4H)-thione}

\author{
Rizvan K. Askerov, ${ }^{a}$ Abel M. Maharramov, ${ }^{a}$ Ali N. Khalilov, ${ }^{\text {a,b }}$ Mehmet Akkurt, \\ Anzurat A. Akobirshoeva, ${ }^{\text {d* V. K. Osmanov }}$ and A. V. Borisov $^{\text {e }}$
}

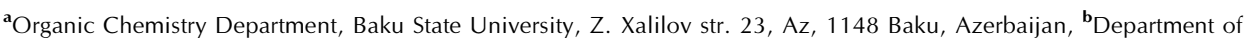
Physics and Chemistry, "Composite Materials" Scientific Research Center, Azerbaijan State Economic University (UNEC), H. Aliyev str. 135, Az 1063, Baku, Azerbaijan, 'Department of Physics, Faculty of Sciences, Erciyes University, 38039 Kayseri, Turkey, ${ }^{\mathbf{d}}$ Acad. Sci. Republ. Tadzhikistan, Kh. Yu. Yusufbekov Pamir Biol. Inst., 1 Kholdorova St, Khorog 736002, Gbao, Tajikistan, and ${ }^{\mathbf{e}}$ Nizhny Novgorod State Technical University n.a. R.E. Alekseev, Nizhny Novgorod, Russian Federation. *Correspondence e-mail: anzurat2003@mail.ru

In the crystal of the title compound, $\mathrm{C}_{7} \mathrm{H}_{5} \mathrm{FN}_{4} \mathrm{~S}$, the molecules are non-planar, with dihedral angle formed by least-squares planes of tetrazole and benzene rings of $59.94(8)^{\circ}$. The crystal packing is formed by $\mathrm{N}-\mathrm{H} \cdots \mathrm{S}$ hydrogen bonds, which link the molecules into centrosymmetric dimers with an $R_{2}^{2}(8)$ ring motif, and by the offset face-to-face $\pi-\pi$ stacking interactions between the benzene rings, which join the dimers into layers parallel to (100). The Hirshfeld surface analysis shows that the most important contributions to the surface contacts are from $\mathrm{N} \cdots \mathrm{H} / \mathrm{H} \cdots \mathrm{N}(21.9 \%), \mathrm{S} \cdots \mathrm{H} / \mathrm{H} \cdots \mathrm{S}(21.1 \%), \mathrm{H} \cdots \mathrm{H}(14.6 \%), \mathrm{F} \cdots \mathrm{H} /$ $\mathrm{H} \cdots \mathrm{F}(11.8 \%)$ and $\mathrm{C} \cdot \mathrm{H} / \mathrm{H} \cdots \mathrm{C}(9.5 \%)$ interactions.

\section{Chemical context}

Tetrazoles as an important class of five-membered heterocyclic compounds have been known for over a hundred years. The most common synthetic approach to construct tetrazoles, based on the reaction of nitriles with hydrazoic acid, was first discovered by Hantzsch \& Vagt (1901). Up to know, most synthetic protocols comprise the cycloaddition of nitriles, thiocyanates or isothiocyanates with an azide moiety, under different conditions. Tetrazole derivatives have found a broad range of applications in medicinal chemistry (Wang et al., 2019; Gao et al., 2019; Arulmozhi et al., 2017), coordination chemistry (Askerov et al., 2018; Askerov et al., 2019a,b; Aromí et al., 2011) and material science (Frija et al., 2010; Lv et al., 2006). Numerous tetrazole-based synthetic compounds such as tomelukast, cefazolin, losartan, valsartan and alfentanil have already been used in medicinal practice.<smiles>Fc1ccccc1-n1nn[nH]c1=S</smiles>

As a result of the considerable interest in this field, significant developments in the synthesis of tetrazoles have been attained, which were recently reviewed (Neochoritis et al., 2019). As a further study of the chemistry of tetrazoles, herein we report the crystal structure and Hirshfeld surface analysis of the title compound. 
Table 1

Hydrogen-bond geometry $\left(\AA,^{\circ}\right)$.

\begin{tabular}{lllll}
\hline$D-\mathrm{H} \cdots A$ & $D-\mathrm{H}$ & $\mathrm{H} \cdots A$ & $D \cdots A$ & $D-\mathrm{H} \cdots A$ \\
\hline $\mathrm{N} 4-\mathrm{H} 4 \cdots \mathrm{S} 1^{\mathrm{i}}$ & $0.90(2)$ & $2.35(2)$ & $3.2456(12)$ & $173.2(18)$ \\
\hline
\end{tabular}

Symmetry code: (i) $-x+1,-y+2,-z+1$.

\section{Structural commentary}

The molecule of the title compound (Fig. 1) is non-planar. The five-membered 4-dihydro-5 $H$-tetrazole ring $(\mathrm{N} 1-\mathrm{N} 4 / \mathrm{C} 5)$ is essentially planar, with a largest deviation of 0.005 (1) $\AA$ for N3. The dihedral angle between the mean planes of the tetrazole and benzene rings is $59.94(8)^{\circ}$. The bond dimensions are typical of similar compounds, with a distinct $\mathrm{N} 2=\mathrm{N} 3$ double bond.

\section{Supramolecular features}

In the crystal, molecules are linked by pairs of $\mathrm{N}-\mathrm{H} \cdots \mathrm{S}$ hydrogen bonds, forming centrosymmetric dimers with an $R_{2}^{2}(8)$ ring motif (see Fig. 2 and Table 1). The dimers are linked by the offset face-to-face $\pi-\pi$ stacking interactions between the benzene rings, which are characterized by intercentroid distances of 3.8963 (9) and 3.8964 (9) $\AA$, and centroid-to-plane distances of 3.4589 (6) and 3.4578 (6) A (Fig. 2). Neighbouring molecules within the stack are related by the $c$ glide plane. The hydrogen bonds and stacking interactions link the molecules into layers parallel to (100). Other short intermolecular contacts are collected in Table 2.

\section{Hirshfeld surface analysis}

In order to investigate the intermolecular interactions in the crystal structure of the title compound in a visual manner, Hirshfeld surfaces (McKinnon et al., 2007) and their associated two-dimensional fingerprint plots (Spackman \& McKinnon, 2002) were generated using CrystalExplorer17 (Turner et al., 2017). The shorter and longer contacts are indicated as red and blue spots, respectively, on the Hirshfeld surfaces, and contacts with distances approximately equal to

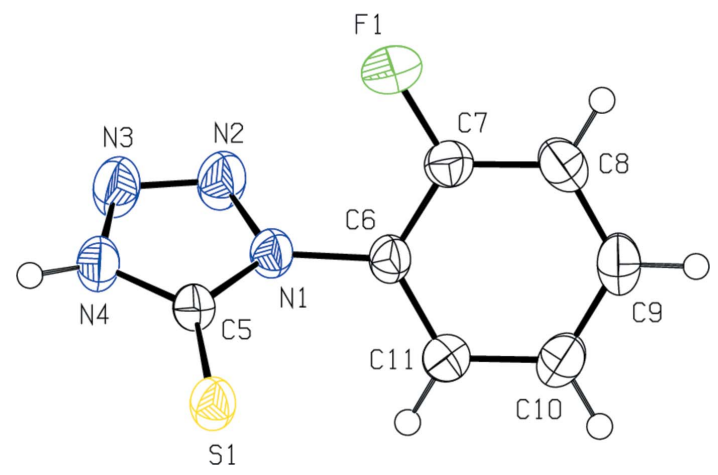

Figure 1

The molecular structure of the title compound, showing displacement ellipsoids drawn at the $50 \%$ probability level.
Table 2

Summary of short interatomic contacts $(\AA)$ in the title compound.

\begin{tabular}{lll}
\hline Contact & Distance & Symmetry operation \\
\hline S1 $\cdots \mathrm{S} 1$ & $3.7741(6)$ & $1-x, y, \frac{3}{2}-z$ \\
C5 $\cdots \mathrm{S} 1$ & $3.6367(13)$ & $1-x, y, \frac{1}{2}-z$ \\
$\mathrm{H} 4 \cdots \mathrm{S} 1$ & 2.35 & $1-x, 2-y, 1-z$ \\
$\mathrm{H} 10 A \cdots \mathrm{S} 1$ & 3.18 & $1-x, 1-y, 1-z$ \\
$\mathrm{~S} 1 \cdots \mathrm{H} 10 A$ & 3.04 & $x, 1-y, \frac{1}{2}+z$ \\
$\mathrm{~F} 1 \cdots \mathrm{H} 8 A$ & 2.63 & $\frac{3}{2}-x, \frac{1}{2}+y, \frac{3}{2}-z$ \\
$\mathrm{~F} 1 \cdots \mathrm{F} 1$ & $3.0330(15)$ & $\frac{3}{2}-x, \frac{3}{2}-y, 1-z$ \\
$\mathrm{~N} 3 \cdots \mathrm{H} 10 A$ & 2.82 & $x, 1+y, z$ \\
$\mathrm{~N} 3 \cdots \mathrm{C} 5$ & 3.38 & $x, 2-y,-\frac{1}{2}+z$ \\
\hline
\end{tabular}

the sum of the van der Waals radii are represented as white spots. The contribution of interatomic contacts (Table 2) to the $d_{\text {norm }}$ surface of the title compound is shown in Fig. 3. In Fig. 4, red and blue triangles can be seen on the shape-index surface, which indicate the presence of $\pi-\pi$ stacking interactions in the crystal structure. Analysis of the two-dimensional fingerprint plots (Fig. 5) reveals that $\mathrm{N} \cdots \mathrm{H} / \mathrm{H} \cdots \mathrm{N}(21.9 \%)$ and $\mathrm{S} \cdots \mathrm{H} /$ $\mathrm{H} \cdots \mathrm{S}(21.1 \%)$ contacts (i.e. $\mathrm{N}-\mathrm{H} \cdots \mathrm{S})$ are the major contri-

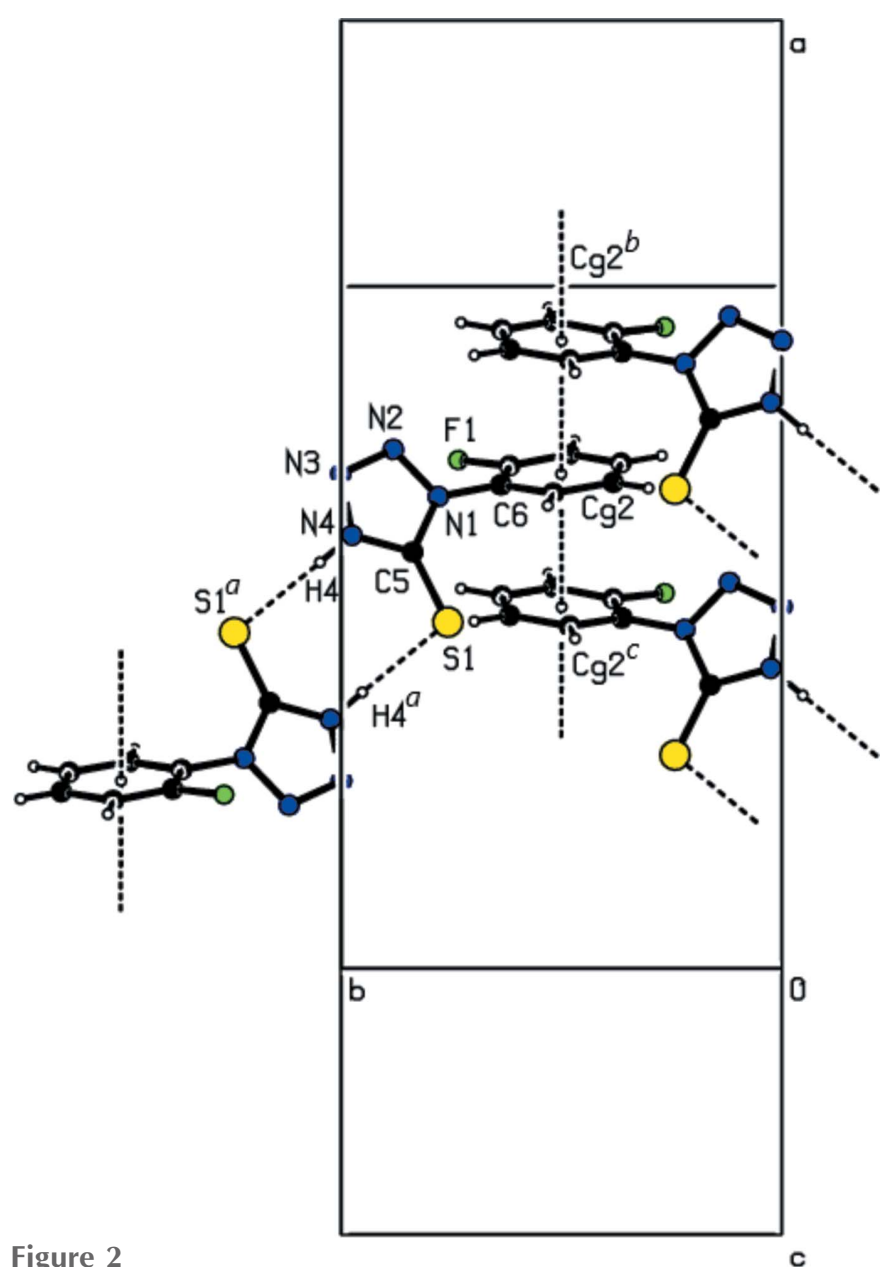

Crystal packing of the title compound viewed along the $a$-axis direction. Dashed lines indicate the $\mathrm{N}-\mathrm{H} \cdots \mathrm{S}$ hydrogen bonds, which form centrosymmetric dimers with an $R_{2}^{2}(8)$ ring motif, and the face-to-face $\pi-\pi$ stacking interactions, which connect the dimers into layers parallel to (100). 


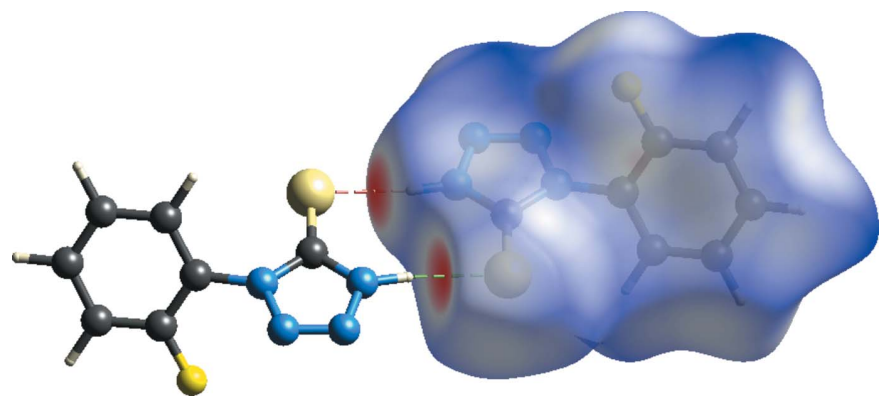

Figure 3

A view of the three-dimensional Hirshfeld surface for the title molecule, plotted over $d_{\text {norm }}$ ranging from -0.4612 to 1.2843 a.u. A dimer formed by $\mathrm{N}-\mathrm{H} \cdots \mathrm{S}$ hydrogen bonds is shown.

butors to the Hirshfeld surface, while $\mathrm{H} \cdots \mathrm{H}(14.6 \%), \mathrm{F} \cdots \mathrm{H} /$ $\mathrm{H} \cdots \mathrm{F}(11.8 \%)$ and $\mathrm{C} \cdots \mathrm{H} / \mathrm{H} \cdots \mathrm{C}(9.5 \%)$ contacts make a less significant contribution. The contribution of the C. . C $(6.6 \%)$ (i.e. $\pi-\pi$ stacking) contacts and other contacts such as $\mathrm{N} \cdots \mathrm{N}$ $(2.8 \%), \mathrm{F} \cdots \mathrm{C} / \mathrm{C} \cdots \mathrm{F}(2.4 \%), \mathrm{N} \cdots \mathrm{C} / \mathrm{C} \cdots \mathrm{N}(2.4 \%), \mathrm{F} \cdots \mathrm{N} /$ N $\cdots \mathrm{F}(1.7 \%), \mathrm{S} \cdots \mathrm{N} / \mathrm{N} \cdots \mathrm{S}(1.7 \%), \mathrm{S} \cdots \mathrm{C} / \mathrm{C} \cdots \mathrm{S}(1.7 \%), \mathrm{F} \cdots \mathrm{F}$ $(1.5 \%)$ and $\mathrm{S} \cdots \mathrm{S}(0.4 \%)$ make a small contribution to the overall Hirshfeld surface.

\section{Database survey}

A search of the Cambridge Crystallographic Database (CSD version 5.40, update of September 2019; Groom et al., 2016) yielded nine entries closely related to the title compound, viz. 1-(4-fluorophenyl)-4,4,6-trimethyl-3,4-dihydropyrimidine2(1H)-thione (CSD refcode ASEHIR; Kadir et al., 2016), 3(adamantan-1-yl)-4-(4-fluorophenyl)-1-[(4-phenylpiperazin-1yl)methyl]-4,5-dihydro-1H-1,2,4-triazole-5-thione (ZEFKED; Al-Alshaikh et al., 2017), 3-(adamantan-1-yl)-4-(4-fluorophenyl)-1-\{[4-(2-methoxyphenyl)piperazin-1-yl]-methyl\}-4,5dihydro-1H-1,2,4-triazole-5-thione (ZEFKAZ; Al-Alshaikh et al., 2017), 3-(adamantan-1-yl)-4-(2-bromo-4-fluorophenyl)-

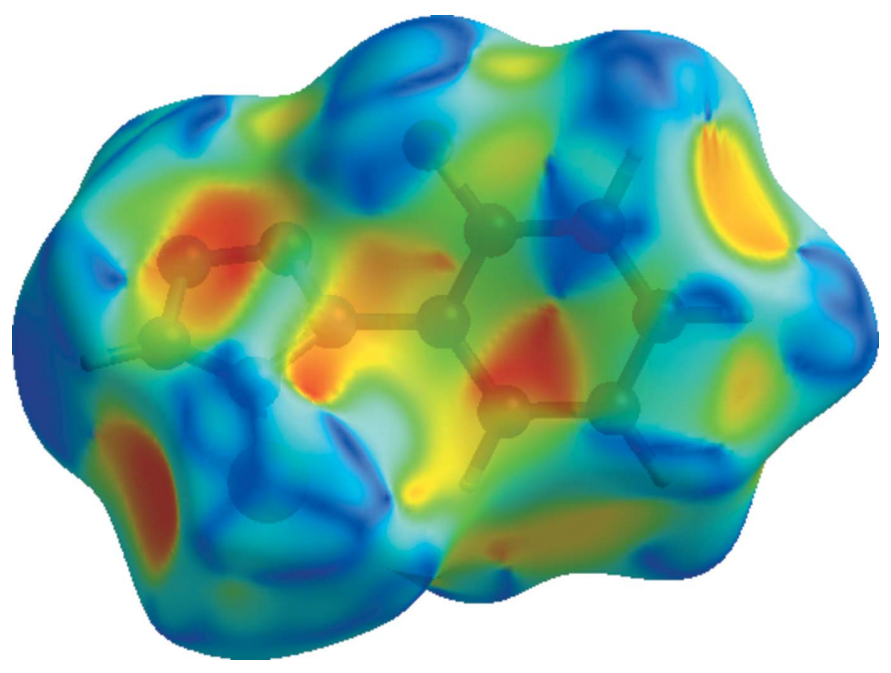

Figure 4

Hirshfeld surface of the title molecule plotted over shape-index.
1H-1,2,4-triazole-5(4H)-thione (ZOZNEK; Abdelrazeq et al., 2020), 2-fluoro- $N$-(3-(methylsulfanyl)-1H-1,2,4-triazol-5-yl)benzamide (MITMOU; Moreno-Fuquen et al., 2019), (5amino-3-(methylsulfanyl)-1H-1,2,4-triazol-1-yl)(2-fluorophenyl)methanone (MITMIO; Moreno-Fuquen et al., 2019), 4(benzo[b]thiophen-2-yl)-5-(3,4,5-trimethoxyphenyl)-2H-1,2,3triazole (PONWIA; Penthala et al., 2014), 4-(benzo[b]thiophen-2-yl)-2-methyl-5-(3,4,5-trimethoxyphenyl)-2H-1,2,3-triazole (PONWOG; Penthala et al., 2014), (E)-3-(4-fluorophenyl)-1-[1-(4-fluorophenyl)-5-methyl-1 $H$-1,2,3-triazol-4-yl]prop2-en-1-one (MESTAI; El-Hiti et al., 2018), 4-amino-3-methyl5-( $p$-tolyl)-4H-1,2,4-triazole (JESTOR; Şahin et al., 2006), 4amino-3-methyl-5-phenyl-4H-1,2,4-triazole (JESTUX; Şahin et al., 2006), and 2-phenyl-4,5-dianilino-2H-1,2,3-triazole (PANTZL10; Harlow et al., 1977).

In the crystal of ASEHIR, pairs of molecules related by the twofold rotation axis are linked by $\mathrm{N}-\mathrm{H} \cdot \mathrm{S}$ hydrogen bonds, forming dimers.

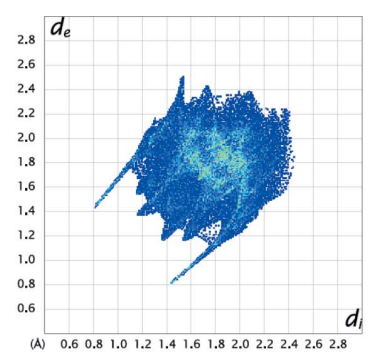

(a) All...All

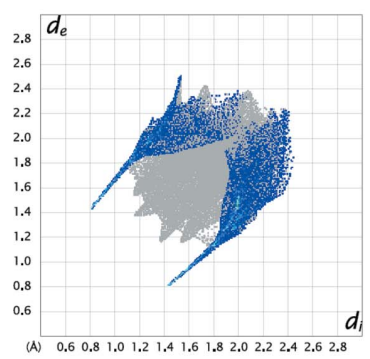

(c) $S \ldots H / H \ldots S$

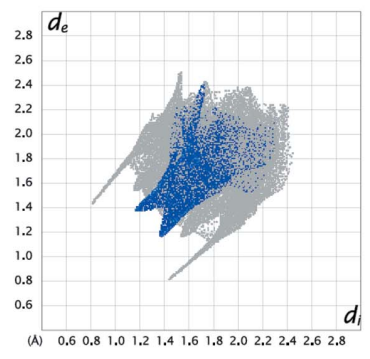

(e) $F \ldots H / H \ldots F$

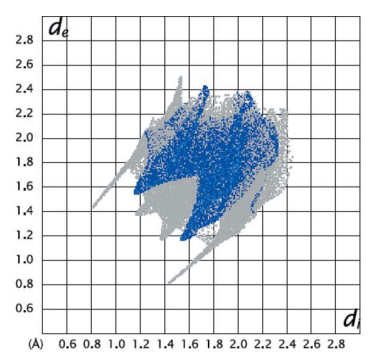

(b) $N \ldots H / H \ldots N$

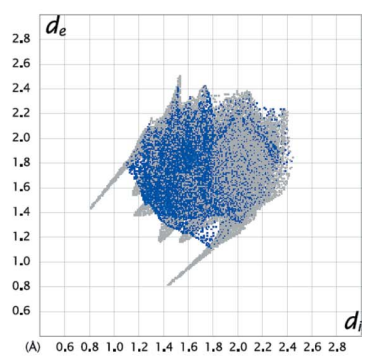

(d) $H$...H

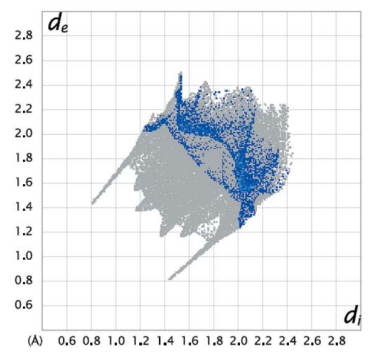

(f) $C$...H $/ H \ldots C$
Figure 5

A view of two-dimensional fingerprint plots for the title compound, showing $(a)$ all interactions, and delineated into $(b) \mathrm{N} \cdots \mathrm{H} / \mathrm{H} \cdots \mathrm{N},(c)$ $\mathrm{S} \cdots \mathrm{H} / \mathrm{H} \cdots \mathrm{S},(d) \mathrm{H} \cdots \mathrm{H},(e) \mathrm{F} \cdots \mathrm{H} / \mathrm{H} \cdots \mathrm{F}$ and $(f) \mathrm{C} \cdots \mathrm{H} / \mathrm{H} \cdots \mathrm{C}$ interactions. The $d_{\mathrm{i}}$ and $d_{\mathrm{e}}$ values are the closest internal and external distances (in $\AA$ ) from given points on the Hirshfeld surface contacts. 
The crystal structure of ZEFKED shows pairs of $\mathrm{C}-\mathrm{H} \cdot \mathrm{F}$ hydrogen bonds forming inversion dimers, while in the crystal of ZEFKAZ, in addition to the $\mathrm{C}-\mathrm{H} \cdots \mathrm{F}$ hydrogen bonds that generate chains parallel to the $b$ axis, there are $\mathrm{C}-\mathrm{H} \cdots \pi$ interactions that link the chains to form layers parallel to the $a b$ plane.

In the crystal of ZOZNEK, the molecules are linked by weak $\mathrm{C}-\mathrm{H} \cdots \pi$ (phenyl) interactions, forming supramolecular chains extending along the $c$-axis direction. The crystal packing is further consolidated by intermolecular $\mathrm{N}-\mathrm{H} \cdots \mathrm{S}$ hydrogen bonds and by weak $\mathrm{C}-\mathrm{H} \cdots \mathrm{S}$ interactions, yielding double chains propagating along the $a$-axis direction.

In the crystal structure of MITMOU, the supramolecular assembly is formed mainly by $(\mathrm{N}, \mathrm{C})-\mathrm{H} \cdots(\mathrm{N}, \mathrm{O})$ hydrogenbond interactions. Initially, strong $\mathrm{N}-\mathrm{H} \cdots \mathrm{N}$ hydrogen bonds link pairs of inversion-related molecules that act as slabs of infinite chains running along the [100] direction connected by a $\mathrm{C}-\mathrm{H} \cdots \mathrm{O}$ hydrogen bond. Along the [010] direction, neighbouring chains are further connected by weak $\pi-\pi$ interactions between two arene rings of adjacent molecules.

The crystal structure of MITMIO is built by a combination of strong $\mathrm{N}-\mathrm{H} \cdots \mathrm{O}$ and $\mathrm{N}-\mathrm{H} \cdots \mathrm{N}$ hydrogen bonds, which form chains of molecules running along the [100] direction. Parallel inversion-related chains of molecules are further connected by weaker $\mathrm{C}-\mathrm{H}$.. O interactions to build the molecular architecture along the [001] direction. Weak $\mathrm{C}-$ $\mathrm{H} \cdots \mathrm{N}$ interactions connect the molecules in order to complete the three-dimensional structure along the [010] direction.

In the crystal of PONWIA, the molecules are linked into chains by $\mathrm{N}-\mathrm{H} \cdots \mathrm{O}$ hydrogen bonds with $R_{1}^{2}(5)$ ring motifs. After the $N$-methylation of the PONWIA molecule, no hydrogen-bonding interactions were observed for structure PONWOG. The crystal structure of PONWOG shows a disorder due to a $180^{\circ}$ flip of the benzothiophene ring system.

In the crystal of MESTAI, the asymmetric unit comprises two molecules with similar conformations. In the crystal, weak $\mathrm{C}-\mathrm{H} \cdots \mathrm{F}$ interactions form chains of molecules and the chains are stacked to form layers parallel to (101).

In JESTOR, molecules are linked principally by $\mathrm{N}-\mathrm{H} \cdots \mathrm{N}$ hydrogen bonds involving the amino $\mathrm{NH}_{2}$ group and a triazole $\mathrm{N}$ atom, forming $R_{4}^{4}(20)$ and $R_{4}^{2}(10)$ rings that combine to give a three-dimensional network of molecules. The hydrogen bonding is supported by two different $\mathrm{C}-\mathrm{H} \cdots \pi$ interactions from the tolyl ring to either a triazole ring or a tolyl ring in a neighboring molecule. In JESTUX, intermolecular hydrogen bonds and $\mathrm{C}-\mathrm{H} \cdots \pi$ interactions generate $R_{4}^{3}(15)$ and $R_{4}^{4}(21)$ rings.

\section{Synthesis and crystallization}

To a solution of of $\mathrm{NaN}_{3}(29 \mathrm{mmol})$ in $50 \mathrm{~mL}$ of $\mathrm{H}_{2} \mathrm{O} 2$ fluorophenylisothiocyanate $(19.6 \mathrm{mmol})$ was added at $293 \mathrm{~K}$. The reaction mixture was boiled for $2 \mathrm{~h}$, cooled to $293 \mathrm{~K}$; then the aqueous solution was filtered from undissolved impurities and a $10 \%$ aqueous solution of $\mathrm{HCl}$ was added to it with stirring to $\mathrm{pH}=2$. The precipitate of the title compound was
Table 3

Experimental details.

\begin{tabular}{|c|c|}
\hline \multicolumn{2}{|l|}{ Crystal data } \\
\hline Chemical formula & $\mathrm{C}_{7} \mathrm{H}_{5} \mathrm{FN}_{4} \mathrm{~S}$ \\
\hline$M_{\mathrm{r}}$ & 196.21 \\
\hline Crystal system, space group & Monoclinic, $C 2 / c$ \\
\hline Temperature $(\mathrm{K})$ & 296 \\
\hline$a, b, c(\AA)$ & $23.5593(11), 9.2849$ (5), 7.7927 (4) \\
\hline$\beta\left(^{\circ}\right)$ & 104.009 (1) \\
\hline$V\left(\AA^{3}\right)$ & $1653.92(15)$ \\
\hline$Z$ & 8 \\
\hline Radiation type & Мo $K \alpha$ \\
\hline$\mu\left(\mathrm{mm}^{-1}\right)$ & 0.36 \\
\hline Crystal size $(\mathrm{mm})$ & $0.23 \times 0.15 \times 0.08$ \\
\hline \multicolumn{2}{|l|}{ Data collection } \\
\hline Diffractometer & Bruker APEXII CCD \\
\hline Absorption correction & $\begin{array}{l}\text { Multi-scan (SADABS; Bruker, } \\
\text { 2003) }\end{array}$ \\
\hline$T_{\min }, T_{\max }$ & $0.690,0.746$ \\
\hline $\begin{array}{l}\text { No. of measured, independent and } \\
\text { observed }[I>2 \sigma(I)] \text { reflections }\end{array}$ & $9005,2406,2135$ \\
\hline$R_{\text {int }}$ & 0.017 \\
\hline$(\sin \theta / \lambda)_{\max }\left(\AA^{-1}\right)$ & 0.713 \\
\hline \multicolumn{2}{|l|}{ Refinement } \\
\hline$R\left[F^{2}>2 \sigma\left(F^{2}\right)\right], w R\left(F^{2}\right), S$ & $0.036,0.098,1.00$ \\
\hline No. of reflections & 2406 \\
\hline No. of parameters & 122 \\
\hline $\mathrm{H}$-atom treatment & $\begin{array}{l}\mathrm{H} \text { atoms treated by a mixture of } \\
\text { independent and constrained } \\
\text { refinement }\end{array}$ \\
\hline$\Delta \rho_{\max }, \Delta \rho_{\min }\left(\mathrm{e} \AA^{-3}\right)$ & $0.29,-0.22$ \\
\hline
\end{tabular}

Computer programs: APEX2 and SAINT (Bruker, 2003), SHELXT2014/5 (Sheldrick, 2015a), SHELXL2018/3 (Sheldrick, 2015b), ORTEP-3 for Windows (Farrugia, 2012) and PLATON (Spek, 2020).

filtered off, washed with water, and then the product was recrystallized from ethanol.

1-(2-Fluorophenyl)-1H-tetrazole-5(4H)-thione: yield $72 \%$ as white powder, m.p. $426 \mathrm{~K}$. Analysis calculated for $\mathrm{C}_{7} \mathrm{H}_{5} \mathrm{FN}_{4} \mathrm{~S}$ (\%): C 42.85, H 2.57, N 28.56. Found (\%): C 42.62, $\mathrm{H} 2.66, \mathrm{~N} 28.59 .{ }^{1} \mathrm{H}$ NMR $\left(400.00 \mathrm{MHz}, \mathrm{DMSO}-d_{6}\right): \delta=7.73$ $(m, 1 \mathrm{H}), 7.68(m, 1 \mathrm{H}), 7.55(t, 1 \mathrm{H}), 7.45(t, 1 \mathrm{H}) .{ }^{13} \mathrm{C} \mathrm{NMR}$ $\left(100.60 \mathrm{MHz}, \mathrm{DMSO}-d_{6}\right): \delta=162.76(\mathbf{C}=\mathrm{S}), 156.39(\mathbf{C}-\mathrm{F})$, [137.33 (C-N, Ar), 128.76, 127.08, 124.94, 114.88 (4CH, Ar)].

\section{Refinement details}

Crystal data, data collection and structure refinement details are summarized in Table 3 . The $\mathrm{C}$-bound $\mathrm{H}$ atoms were placed in calculated positions $(0.93 \AA)$ and refined as riding with $U_{\text {iso }}(\mathrm{H})=1.2 U_{\text {eq }}(\mathrm{C})$. The $\mathrm{N}$-bound $\mathrm{H}$ atom was located in a difference map and refined isotropically.

\section{References}

Abdelrazeq, A. S., Ghabbour, H. A., El-Emam, A. A., Osman, D. A. \& Garcia-Granda, S. (2020). Acta Cryst. E76, 162-166.

Al-Alshaikh, M. A., Al-Mutairi, A. A., Ghabbour, H. A., El-Emam, A. A., Abdelbaky, M. S. M. \& Garcia-Granda, S. (2017). Acta Cryst. E73, 1135-1139.

Aromí, G., Barrios, L. A., Roubeau, O. \& Gamez, P. (2011). Coord. Chem. Rev. 255, 485-546.

Arulmozhi, R., Abirami, N. \& Helen, K. P. (2017). Int. J. Pharm. Sci. Rev. Res. 21, 110-114. 
Askerov, R. K., Magerramov, A. M., Osmanov, V. K., Baranov, E. V., Borisova, G. N. \& Borisov, A. V. (2019b). Russ. J. Coord. Chem. 45, $555-562$.

Askerov, R. K., Magerramov, A. M., Osmanov, V. K., Baranov, E. V., Borisova, G. N., Samsonova, A. D. \& Borisov, A. V. (2019a). Russ. J. Coord. Chem. 45, 112-118.

Askerov, R. K., Maharramov, A. M., Osmanov, V. K., Baranov, E. V., Borisova, G. N., Dorovatovskii, P. V., Khrustalev, V. N. \& Borisov, A. V. (2018). J. Struct. Chem. 59, 1658-1663.

Bruker (2003). APEX2, SAINT and SADABS. Bruker AXS Inc., Madison, Wisconsin, USA.

El-Hiti, G. A., Abdel-Wahab, B. F., Alotaibi, M. H., Hegazy, A. S. \& Kariuki, B. M. (2018). IUCrData, 3, x171841.

Farrugia, L. J. (2012). J. Appl. Cryst. 45, 849-854.

Frija, L. M. T., Ismael, A. \& Cristiano, M. L. S. (2010). Molecules, 15, 3757-3774.

Gao, C., Chang, L., Xu, Z., Yan, X.-F., Ding, C., Zhao, F., Wu, X. \& Feng, L.-S. (2019). Eur. J. Med. Chem. 163, 404-412.

Groom, C. R., Bruno, I. J., Lightfoot, M. P. \& Ward, S. C. (2016). Acta Cryst. B72, 171-179.

Hantzsch, A. \& Vagt, A. (1901). Justus Liebigs Ann. Chem. 314, 339369.

Harlow, R. L., Brown, S. B., Dewar, M. J. S. \& Simonsen, S. H. (1977). Acta Cryst. B33, 3423-3428.
Kadir, A., Abd Malek, N. A., Mohd Zaki, H., Hasbullah, S. A. \& Yamin, B. M. (2016). IUCrData, 1, x161189.

Lv, F., Liu, Y., Zou, J., Zhang, D. \& Yao, Z. (2006). Dyes Pigments, 68 , 211-216.

McKinnon, J. J., Jayatilaka, D. \& Spackman, M. A. (2007). Chem. Commun. pp. 3814-3816.

Moreno-Fuquen, R., Arango-Daraviña, K., Becerra, D., Castillo, J.-C., Kennedy, A. R. \& Macías, M. A. (2019). Acta Cryst. C75, 359371.

Neochoritis, C. G., Zhao, T. \& Dömling, A. (2019). Chem. Rev. 119, 1970-2042.

Penthala, N. R., Madadi, N. R., Bommagani, S., Parkin, S. \& Crooks, P. A. (2014). Acta Cryst. E70, 392-395.

Şahin, O., Büyükgüngör, O., Şaşmaz, S., Gümrükçüoğlu, N. \& Kantar, C. (2006). Acta Cryst. C62, o643-o646.

Sheldrick, G. M. (2015a). Acta Cryst. A71, 3-8.

Sheldrick, G. M. (2015b). Acta Cryst. C71, 3-8.

Spackman, M. A. \& McKinnon, J. J. (2002). CrystEngComm, 4, 378392.

Spek, A. L. (2020). Acta Cryst. E76, 1-11.

Turner, M. J., Mckinnon, J. J., Wolff, S. K., Grimwood, D. J., Spackman, P. R., Jayatilaka, D. \& Spackman, M. A. (2017). CrystalExplorer17. The University of Western Australia.

Wang, S.-Q., Wang, Y.-F. \& Xu, Z. (2019). Eur. J. Med. Chem. 170, 225-234. 


\section{supporting information}

Acta Cryst. (2020). E76, 1007-1011 [https://doi.org/10.1107/S2056989020007033]

\section{Crystal structure and Hirshfeld surface analysis of 1-(2-fluorophenyl)-1H-}

\section{tetrazole-5(4H)-thione}

\section{Rizvan K. Askerov, Abel M. Maharramov, Ali N. Khalilov, Mehmet Akkurt, Anzurat A.}

Akobirshoeva, V. K. Osmanov and A. V. Borisov

\section{Computing details}

Data collection: APEX2 (Bruker, 2003); cell refinement: SAINT (Bruker, 2003); data reduction: SAINT (Bruker, 2003); program(s) used to solve structure: SHELXT2014/5 (Sheldrick, 2015a); program(s) used to refine structure:

SHELXL2018/3 (Sheldrick, 2015b); molecular graphics: ORTEP-3 for Windows (Farrugia, 2012); software used to prepare material for publication: PLATON (Spek, 2020).

1-(2-Fluorophenyl)-1H-tetrazole-5(4H)-thione

Crystal data

$\mathrm{C}_{7} \mathrm{H}_{5} \mathrm{FN}_{4} \mathrm{~S}$

$M_{r}=196.21$

Monoclinic, $C 2 / c$

$a=23.5593(11) \AA$

$b=9.2849(5) \AA$

$c=7.7927(4) \AA$

$\beta=104.009(1)^{\circ}$

$V=1653.92(15) \AA^{3}$

$Z=8$

\section{Data collection}

Bruker APEXII CCD diffractometer

$\varphi$ and $\omega$ scans

Absorption correction: multi-scan

(SADABS; Bruker, 2003)

$T_{\text {min }}=0.690, T_{\max }=0.746$

9005 measured reflections

\section{Refinement}

Refinement on $F^{2}$

Least-squares matrix: full

$R\left[F^{2}>2 \sigma\left(F^{2}\right)\right]=0.036$

$w R\left(F^{2}\right)=0.098$

$S=1.00$

2406 reflections

122 parameters

0 restraints
$F(000)=800$

$D_{\mathrm{x}}=1.576 \mathrm{Mg} \mathrm{m}^{-3}$

Mo $K \alpha$ radiation, $\lambda=0.71073 \AA$

Cell parameters from 4417 reflections

$\theta=2.4-30.5^{\circ}$

$\mu=0.36 \mathrm{~mm}^{-1}$

$T=296 \mathrm{~K}$

Prism, colourless

$0.23 \times 0.15 \times 0.08 \mathrm{~mm}$

2406 independent reflections

2135 reflections with $I>2 \sigma(I)$

$R_{\text {int }}=0.017$

$\theta_{\max }=30.5^{\circ}, \theta_{\min }=1.8^{\circ}$

$h=-32 \rightarrow 33$

$k=-13 \rightarrow 13$

$l=-10 \rightarrow 10$

Primary atom site location: difference Fourier map

Secondary atom site location: difference Fourier map

Hydrogen site location: mixed

$\mathrm{H}$ atoms treated by a mixture of independent and constrained refinement 
$w=1 /\left[\sigma^{2}\left(F_{\mathrm{o}}^{2}\right)+(0.0487 P)^{2}+1.1614 P\right]$

where $P=\left(F_{\mathrm{o}}^{2}+2 F_{\mathrm{c}}^{2}\right) / 3$

$(\Delta / \sigma)_{\max }=0.001$

$$
\Delta \rho_{\max }=0.29 \mathrm{e} \AA^{-3}
$$

\section{Special details}

Geometry. All esds (except the esd in the dihedral angle between two 1.s. planes) are estimated using the full covariance matrix. The cell esds are taken into account individually in the estimation of esds in distances, angles and torsion angles; correlations between esds in cell parameters are only used when they are defined by crystal symmetry. An approximate (isotropic) treatment of cell esds is used for estimating esds involving 1.s. planes.

Fractional atomic coordinates and isotropic or equivalent isotropic displacement parameters $\left(\AA^{2}\right)$

\begin{tabular}{lllll}
\hline & $x$ & $y$ & $z$ & $U_{\text {iso }} * / U_{\text {eq }}$ \\
\hline S1 & $0.51038(2)$ & $0.75776(4)$ & $0.51736(5)$ & $0.03900(12)$ \\
F1 & $0.71634(4)$ & $0.73413(10)$ & $0.64199(14)$ & $0.0536(3)$ \\
$\mathrm{N} 1$ & $0.61196(5)$ & $0.77955(12)$ & $0.40738(16)$ & $0.0346(2)$ \\
$\mathrm{N} 2$ & $0.64339(6)$ & $0.88176(13)$ & $0.3424(2)$ & $0.0480(3)$ \\
$\mathrm{N} 3$ & $0.61568(6)$ & $1.00050(13)$ & $0.3346(2)$ & $0.0494(3)$ \\
$\mathrm{N} 4$ & $0.56649(5)$ & $0.97577(12)$ & $0.39123(17)$ & $0.0399(3)$ \\
$\mathrm{H} 4$ & $0.5428(9)$ & $1.048(2)$ & $0.408(3)$ & $0.060(5)^{*}$ \\
C5 & $0.56255(5)$ & $0.83768(13)$ & $0.43970(17)$ & $0.0320(2)$ \\
C6 & $0.63424(5)$ & $0.63719(13)$ & $0.44289(17)$ & $0.0314(2)$ \\
C7 & $0.68772(6)$ & $0.61740(14)$ & $0.56178(18)$ & $0.0361(3)$ \\
C8 & $0.71115(6)$ & $0.48238(16)$ & $0.6014(2)$ & $0.0434(3)$ \\
H8A & 0.747433 & 0.470495 & 0.680048 & $0.052^{*}$ \\
C9 & $0.67955(7)$ & $0.36465(16)$ & $0.5217(2)$ & $0.0444(3)$ \\
H9A & 0.694563 & 0.272435 & 0.547932 & $0.053^{*}$ \\
C10 & $0.62584(7)$ & $0.38250(15)$ & $0.4034(2)$ & $0.0419(3)$ \\
H10A & 0.604979 & 0.302279 & 0.351172 & $0.050^{*}$ \\
C11 & $0.60297(6)$ & $0.51874(14)$ & $0.36219(19)$ & $0.0369(3)$ \\
H11A & 0.567110 & 0.530830 & 0.281472 & $0.044 *$ \\
& & & &
\end{tabular}

Atomic displacement parameters $\left(\AA^{2}\right)$

\begin{tabular}{lllllll}
\hline & $U^{11}$ & $U^{22}$ & $U^{33}$ & $U^{12}$ & $U^{13}$ & $U^{23}$ \\
\hline S1 & $0.03642(19)$ & $0.03299(18)$ & $0.0519(2)$ & $0.00355(12)$ & $0.01903(15)$ & $0.00288(13)$ \\
F1 & $0.0467(5)$ & $0.0476(5)$ & $0.0603(6)$ & $-0.0097(4)$ & $0.0012(4)$ & $-0.0104(4)$ \\
N1 & $0.0316(5)$ & $0.0285(5)$ & $0.0465(6)$ & $0.0026(4)$ & $0.0148(4)$ & $0.0020(4)$ \\
N2 & $0.0431(6)$ & $0.0347(6)$ & $0.0728(9)$ & $0.0013(5)$ & $0.0267(6)$ & $0.0084(6)$ \\
N3 & $0.0476(7)$ & $0.0330(6)$ & $0.0737(9)$ & $0.0031(5)$ & $0.0266(6)$ & $0.0085(6)$ \\
N4 & $0.0404(6)$ & $0.0291(5)$ & $0.0529(7)$ & $0.0056(4)$ & $0.0165(5)$ & $0.0035(5)$ \\
C5 & $0.0314(5)$ & $0.0287(6)$ & $0.0356(6)$ & $0.0034(4)$ & $0.0076(5)$ & $-0.0012(4)$ \\
C6 & $0.0311(5)$ & $0.0277(5)$ & $0.0378(6)$ & $0.0035(4)$ & $0.0126(5)$ & $0.0009(4)$ \\
C7 & $0.0334(6)$ & $0.0351(6)$ & $0.0400(6)$ & $-0.0017(5)$ & $0.0095(5)$ & $-0.0028(5)$ \\
C8 & $0.0350(6)$ & $0.0463(8)$ & $0.0475(8)$ & $0.0082(6)$ & $0.0072(6)$ & $0.0077(6)$ \\
C9 & $0.0486(8)$ & $0.0338(7)$ & $0.0548(8)$ & $0.0104(6)$ & $0.0202(6)$ & $0.0088(6)$ \\
C10 & $0.0472(7)$ & $0.0307(6)$ & $0.0508(8)$ & $-0.0028(5)$ & $0.0178(6)$ & $-0.0032(6)$ \\
C11 & $0.0345(6)$ & $0.0338(6)$ & $0.0421(7)$ & $-0.0007(5)$ & $0.0086(5)$ & $-0.0014(5)$ \\
\hline
\end{tabular}


Geometric parameters $(\AA, \stackrel{\circ}{)})$

\begin{tabular}{|c|c|c|c|}
\hline $\mathrm{S} 1-\mathrm{C} 5$ & $1.6696(13)$ & $\mathrm{C} 6-\mathrm{C} 11$ & $1.3868(18)$ \\
\hline $\mathrm{F} 1-\mathrm{C} 7$ & $1.3476(15)$ & $\mathrm{C} 7-\mathrm{C} 8$ & $1.3745(19)$ \\
\hline $\mathrm{N} 1-\mathrm{C} 5$ & $1.3608(15)$ & $\mathrm{C} 8-\mathrm{C} 9$ & $1.382(2)$ \\
\hline $\mathrm{N} 1-\mathrm{N} 2$ & $1.3735(16)$ & $\mathrm{C} 8-\mathrm{H} 8 \mathrm{~A}$ & 0.9300 \\
\hline $\mathrm{N} 1-\mathrm{C} 6$ & $1.4244(16)$ & $\mathrm{C} 9-\mathrm{C} 10$ & $1.384(2)$ \\
\hline $\mathrm{N} 2-\mathrm{N} 3$ & $1.2752(16)$ & C9-H9A & 0.9300 \\
\hline $\mathrm{N} 3-\mathrm{N} 4$ & $1.3558(17)$ & $\mathrm{C} 10-\mathrm{C} 11$ & $1.3818(19)$ \\
\hline $\mathrm{N} 4-\mathrm{C} 5$ & $1.3462(17)$ & $\mathrm{C} 10-\mathrm{H} 10 \mathrm{~A}$ & 0.9300 \\
\hline $\mathrm{N} 4-\mathrm{H} 4$ & $0.90(2)$ & $\mathrm{C} 11-\mathrm{H} 11 \mathrm{~A}$ & 0.9300 \\
\hline $\mathrm{C} 6-\mathrm{C} 7$ & $1.3836(18)$ & & \\
\hline $\mathrm{C} 5-\mathrm{N} 1-\mathrm{N} 2$ & $110.84(11)$ & $\mathrm{F} 1-\mathrm{C} 7-\mathrm{C} 6$ & $118.43(12)$ \\
\hline $\mathrm{C} 5-\mathrm{N} 1-\mathrm{C} 6$ & $128.58(11)$ & $\mathrm{C} 8-\mathrm{C} 7-\mathrm{C} 6$ & $121.51(12)$ \\
\hline $\mathrm{N} 2-\mathrm{N} 1-\mathrm{C} 6$ & $120.44(10)$ & $\mathrm{C} 7-\mathrm{C} 8-\mathrm{C} 9$ & $118.49(13)$ \\
\hline $\mathrm{N} 3-\mathrm{N} 2-\mathrm{N} 1$ & $107.44(11)$ & $\mathrm{C} 7-\mathrm{C} 8-\mathrm{H} 8 \mathrm{~A}$ & 120.8 \\
\hline $\mathrm{N} 2-\mathrm{N} 3-\mathrm{N} 4$ & $107.81(11)$ & $\mathrm{C} 9-\mathrm{C} 8-\mathrm{H} 8 \mathrm{~A}$ & 120.8 \\
\hline $\mathrm{C} 5-\mathrm{N} 4-\mathrm{N} 3$ & $112.04(11)$ & $\mathrm{C} 8-\mathrm{C} 9-\mathrm{C} 10$ & $120.72(13)$ \\
\hline $\mathrm{C} 5-\mathrm{N} 4-\mathrm{H} 4$ & $125.3(13)$ & $\mathrm{C} 8-\mathrm{C} 9-\mathrm{H} 9 \mathrm{~A}$ & 119.6 \\
\hline $\mathrm{N} 3-\mathrm{N} 4-\mathrm{H} 4$ & $122.1(13)$ & $\mathrm{C} 10-\mathrm{C} 9-\mathrm{H} 9 \mathrm{~A}$ & 119.6 \\
\hline $\mathrm{N} 4-\mathrm{C} 5-\mathrm{N} 1$ & $101.86(11)$ & $\mathrm{C} 11-\mathrm{C} 10-\mathrm{C} 9$ & $120.44(13)$ \\
\hline $\mathrm{N} 4-\mathrm{C} 5-\mathrm{S} 1$ & $129.19(10)$ & $\mathrm{C} 11-\mathrm{C} 10-\mathrm{H} 10 \mathrm{~A}$ & 119.8 \\
\hline $\mathrm{N} 1-\mathrm{C} 5-\mathrm{S} 1$ & $128.94(10)$ & $\mathrm{C} 9-\mathrm{C} 10-\mathrm{H} 10 \mathrm{~A}$ & 119.8 \\
\hline $\mathrm{C} 7-\mathrm{C} 6-\mathrm{C} 11$ & $119.73(12)$ & $\mathrm{C} 10-\mathrm{C} 11-\mathrm{C} 6$ & $119.10(13)$ \\
\hline $\mathrm{C} 7-\mathrm{C} 6-\mathrm{N} 1$ & $119.10(11)$ & $\mathrm{C} 10-\mathrm{C} 11-\mathrm{H} 11 \mathrm{~A}$ & 120.4 \\
\hline $\mathrm{C} 11-\mathrm{C} 6-\mathrm{N} 1$ & $121.17(11)$ & $\mathrm{C} 6-\mathrm{C} 11-\mathrm{H} 11 \mathrm{~A}$ & 120.4 \\
\hline $\mathrm{F} 1-\mathrm{C} 7-\mathrm{C} 8$ & $120.05(12)$ & & \\
\hline $\mathrm{C} 5-\mathrm{N} 1-\mathrm{N} 2-\mathrm{N} 3$ & $0.45(18)$ & $\mathrm{N} 2-\mathrm{N} 1-\mathrm{C} 6-\mathrm{C} 11$ & $-122.21(15)$ \\
\hline $\mathrm{C} 6-\mathrm{N} 1-\mathrm{N} 2-\mathrm{N} 3$ & $-175.58(13)$ & $\mathrm{C} 11-\mathrm{C} 6-\mathrm{C} 7-\mathrm{F} 1$ & $-178.12(12)$ \\
\hline $\mathrm{N} 1-\mathrm{N} 2-\mathrm{N} 3-\mathrm{N} 4$ & $-0.81(18)$ & $\mathrm{N} 1-\mathrm{C} 6-\mathrm{C} 7-\mathrm{F} 1$ & $1.09(18)$ \\
\hline $\mathrm{N} 2-\mathrm{N} 3-\mathrm{N} 4-\mathrm{C} 5$ & $0.94(19)$ & $\mathrm{C} 11-\mathrm{C} 6-\mathrm{C} 7-\mathrm{C} 8$ & $0.5(2)$ \\
\hline $\mathrm{N} 3-\mathrm{N} 4-\mathrm{C} 5-\mathrm{N} 1$ & $-0.62(16)$ & $\mathrm{N} 1-\mathrm{C} 6-\mathrm{C} 7-\mathrm{C} 8$ & $179.75(13)$ \\
\hline $\mathrm{N} 3-\mathrm{N} 4-\mathrm{C} 5-\mathrm{S} 1$ & $179.93(11)$ & $\mathrm{F} 1-\mathrm{C} 7-\mathrm{C} 8-\mathrm{C} 9$ & $177.56(13)$ \\
\hline $\mathrm{N} 2-\mathrm{N} 1-\mathrm{C} 5-\mathrm{N} 4$ & $0.11(15)$ & $\mathrm{C} 6-\mathrm{C} 7-\mathrm{C} 8-\mathrm{C} 9$ & $-1.1(2)$ \\
\hline $\mathrm{C} 6-\mathrm{N} 1-\mathrm{C} 5-\mathrm{N} 4$ & $175.73(13)$ & $\mathrm{C} 7-\mathrm{C} 8-\mathrm{C} 9-\mathrm{C} 10$ & $0.7(2)$ \\
\hline $\mathrm{N} 2-\mathrm{N} 1-\mathrm{C} 5-\mathrm{S} 1$ & $179.57(11)$ & $\mathrm{C} 8-\mathrm{C} 9-\mathrm{C} 10-\mathrm{C} 11$ & $0.3(2)$ \\
\hline $\mathrm{C} 6-\mathrm{N} 1-\mathrm{C} 5-\mathrm{S} 1$ & $-4.8(2)$ & $\mathrm{C} 9-\mathrm{C} 10-\mathrm{C} 11-\mathrm{C} 6$ & $-0.9(2)$ \\
\hline $\mathrm{C} 5-\mathrm{N} 1-\mathrm{C} 6-\mathrm{C} 7$ & $-116.67(15)$ & $\mathrm{C} 7-\mathrm{C} 6-\mathrm{C} 11-\mathrm{C} 10$ & $0.4(2)$ \\
\hline $\mathrm{N} 2-\mathrm{N} 1-\mathrm{C} 6-\mathrm{C} 7$ & $58.59(18)$ & $\mathrm{N} 1-\mathrm{C} 6-\mathrm{C} 11-\mathrm{C} 10$ & $-178.76(12)$ \\
\hline $\mathrm{C} 5-\mathrm{N} 1-\mathrm{C} 6-\mathrm{C} 11$ & $62.53(19)$ & & \\
\hline
\end{tabular}

Hydrogen-bond geometry $\left(\AA,{ }^{\circ}\right)$

\begin{tabular}{lllll}
\hline$D-\mathrm{H} \cdots A$ & $D-\mathrm{H}$ & $\mathrm{H} \cdots A$ & $D \cdots A$ & $D-\mathrm{H} \cdots A$ \\
\hline $\mathrm{N} 4-\mathrm{H} 4 \cdots \mathrm{S}^{\mathrm{i}}$ & $0.90(2)$ & $2.35(2)$ & $3.2456(12)$ & $173.2(18)$
\end{tabular}


Symmetry codes: (i) $-x+1,-y+2,-z+1$; (ii) $-x+1, y,-z+1 / 2$. 